Available online at www.sciencedirect.com

\section{ScienceDirect}

Journal of Adolescence xx (2008) 1-12
Journal of Adolescence

ELSEVIER

Journal of Adolescence xx (2008) $1-12$

www.elsevier.com/locate/jado

\title{
A longitudinal examination of the influence of maturation on physical self-perceptions and the relationship with physical activity in early adolescent girls
}

\author{
Ann-Marie Knowles*, Ailsa G. Niven, Samantha G. Fawkner, Joan M. Henretty \\ School of Life Sciences, Heriot Watt University, John Muir Building, Riccarton, Edinburgh, \\ Scotland EH14 $4 A S, U K$
}

\begin{abstract}
This longitudinal study investigated the influence of maturation on physical self-perceptions and the relationship with physical activity in early adolescent girls $(N=150$; mean age $=12.79 \pm 0.31)$. Physical characteristics were measured and participants completed the Physical Activity Questionnaire for Children, the Children and Youth Physical Self-Perception Profile and the Pubertal Development Scale on two occasions 12 months apart. The results demonstrated a decrease in overall physical activity levels over 12 months which was not influenced by maturational status or physical characteristics. Additional analysis indicated that physical self-perceptions partially accounted for the explained variance in physical activity change, with physical condition being an important individual predictor of physical activity. Further analysis indicated that body mass was an important individual predictor of changes in perceptions of body attractiveness and physical self-worth. At this age maturation has a limited influence on the physical activity behaviours of early adolescent girls and although the variance in physical activity was partly accounted for by physical self-perceptions, this was a relatively small contribution and other factors related to this drop in physical activity needs to be considered longitudinally.

(C) 2008 Published by Elsevier Ltd on behalf of The Association for Professionals in Services for Adolescents.
\end{abstract}

Keywords: Physical activity; Adolescence; Maturation; Physical self-perceptions

\footnotetext{
* Corresponding author. Tel: +44 (0) 131451 3315; fax: +44 (0) 131451.

E-mail addresses: a.knowles@hw.ac.uk (A.-M. Knowles), a.niven@hw.ac.uk (A.G. Niven), s.g.fawkner@hw.ac.uk (S.G. Fawkner), j.m.henretty@hw.ac.uk (J.M. Henretty).

0140-1971/\$30.00 @ 2008 Published by Elsevier Ltd on behalf of The Association for Professionals in Services for Adolescents. doi:10.1016/j.adolescence.2008.06.001
} 


\section{Introduction}

Physical activity participation has a range of health benefits for children and adolescents, including healthy musculoskeletal growth and development, maintenance of energy balance, psychological well-being and social interaction, as well as preventing future morbidity (Department of Health, 2004). Despite this, adolescence has been identified as a risk period for physical activity attrition, particularly among girls (Grunbaum et al., 2004). Numerous studies have highlighted this decrease in physical activity in adolescent girls in Western populations (Aaron, Storti, Robertson, Kriska \& LaPorte, 2002; Kimm et al., 2002; Nelson, Neumark-Stzainer, Hannan, Sirard \& Story, 2006) with findings from the Scottish Health Survey (Bromley et al., 2003) indicating that the percentage of girls meeting current physical activity guidelines declined from $58 \%$ at age $11-12$ years of age to $41 \%$ at age $13-15$ years.

This drop in physical activity in adolescent girls is coincident with maturation, which usually occurs around the age of 12-13 years, yet few studies have examined the direct influence of maturation on physical activity. Early research by Armstrong, Balding, Gentle and Kirby (1990) suggested that maturation assessed by tanner staging had no influence on sustained periods of physical activity in both boys and girls. Contradictory to these early findings, research by Thompson, Baxter-Jones, Mirwald and Bailey (2003) has provided preliminary evidence of a possible influence of pubertal development on physical activity behaviour. More recently, Baker, Birch, Trost and Davison (2007) identified that early-maturing girls, relative to their peers at age 11 , had significantly lower physical activity levels at age 13 years compared to their latermaturing peers, suggesting that early maturation relative to peers may lead to a decline in physical activity among adolescent girls.

The physical changes that accompany maturation are characterised by an increase in fat mass by approximately $22 \%$ which is not matched by an increase in muscle mass or skeletal tissue (Malina, Bouchard \& Bar-Or, 2004), along with changes in body shape and size that are generally opposed to physical activity participation. Therefore, these physical changes may directly impact on girls' ability to participate in physical activity, for example breast development may directly reduce spontaneous physical activity because of the need for appropriate clothing (Baker et al., 2007). In addition, this natural increase in body fat can result in body changes that are opposed to competence in certain activities. For example individuals with excess body fat may find participation in gymnastics difficult. As a result, as girls mature they may self-select out of sport and physical activity because of competency-related issues (Malina et al., 2004).

Self-perceptions refer to all types of self-referent statements about the self, from those that are global to those that are specific in content (Fox, 1997) and a number of researchers have acknowledged developmental changes in adolescents' self-perceptions as they mature (Harter, 1999; Horn, 2004). In addition, the physical changes during maturation can also lead to an increase in the salience of physique to adolescents' self-perceptions (Smith, 2004), possibly resulting in less positive self-perceptions in the physical domain. Therefore, as girls mature they may perceive themselves to have less positive physical self-perceptions (Crocker, Eklund, \& Kowalski, 2000; Crocker et al., 2003). Research examining the relationship between the physical characteristics of maturation and physical self-perceptions has consistently shown perceptions of body attractiveness to be negatively associated with both percentage body fat (Welk \& Eklund, 2005) and Body 
Mass Index (BMI) (Crocker et al., 2003; Crocker, Sabiston, Kowalski, McDonough, \& Kowalski, 2006; Raustorp, Stahle, Gudasic, Kinnunen, \& Mattsson, 2005; Welk \& Eklund, 2005). This tentatively suggests that the physical changes associated with maturation may influence physical self-perceptions. However, more recently, Davison, Werder, Trost, Baker and Birch (2007) examined the influence of early maturation on physical activity and psychological well-being in 11 year old girls over 2 years. Their findings suggested that maturation had a limited influence on aspects of the physical self in relation to body esteem and perceived athletic competence. These inconsistent findings from few studies highlight the need for further research examining the influence of maturation on physical self-perceptions.

Physical self-perceptions have been linked to a number of motivation theories for being physically active. More specifically, Harter's (1982) Competence Motivation theory suggests that individuals who feel competent in the physical domain will be more likely to participate in physical activity. Weiss and Horn (1990) and Weiss, Bredemeier and Shewchuck (1986) have advocated this theory as being particularly appropriate in the research of children and adolescents' physical activity behaviour because it incorporates developmental changes in perceptions. Research has consistently shown a positive relationship between physical activity and physical self-perceptions (Crocker et al., 2000, 2006; Welk \& Eklund, 2005) with the physical self-perception subscales of sport competence and physical condition being shown to be important positive correlates of physical activity in adolescent girls (Biddle, Whitehead, O'Donovan, \& Nevill, 2005). However, the research examining physical self-perceptions and physical activity is predominantly crosssectional in nature, therefore causality cannot be determined (Crocker et al., 2000, 2006; Biddle et al., 2005; Welk \& Eklund, 2005).

Recent advancements in longitudinal research examining the physical self and physical activity have been made by Crocker et al. $(2003,2006)$. Longitudinal analysis identified that $12.9 \%$ of the decrease in physical activity over 3 years in adolescent girls aged 14-17 years was accounted for by the change in physical self-perceptions (Crocker et al., 2006). More specifically, perceptions of sport competence and physical condition were both cross-sectional correlates and longitudinal predictors of physical activity with physical condition being a significant individual predictor of physical activity. The co-variation between activity change and physical self-perceptions provides support for the contention that the physical self plays an important role in the adoption and maintenance of physical activity (Sonstroem, 1997). However, from their own research findings, Crocker et al. (2006) suggested that a focus on early adolescent girls may be more appropriate in understanding physical activity behaviour because physical self-perceptions became relatively stable during mid to late adolescence. Research examining physical self-perceptions during early adolescence is somewhat sparse and during this time there is also the documented maturity-related decrease in physical activity in adolescent girls.

Therefore, this study aimed to examine the changes in physical self-perceptions and physical activity in early adolescent girls over a 12-month period and identify how these changes were influenced by maturation and physical characteristics. Specifically the aims were (i) to examine the changes in physical activity, maturation, physical characteristics and physical self-perceptions over a 12-month period; (ii) to examine the influence of maturation, physical characteristics and physical self-perceptions on the change in physical activity and (iii) to examine the influence of maturation and physical characteristics on the change in physical self-perceptions. 


\section{Method}

\section{Participants}

Phase 1 data (baseline) was collected from 204 adolescent girls (mean age $11.83 \pm 0.39$ ) from 17 Edinburgh primary schools, representing a range of socio-economic backgrounds. Twelve months later (phase 2), 150 participated in the data collection (mean age $=12.79 \pm 0.31$ ) which took place in six Edinburgh Secondary Schools, therefore tracking the girls from primary school into secondary school. The notable high attrition rate was due to 30 participants moving onto secondary schools out with the present study; 21 participants being absent on the day of data collection and three participants withdrawing without reason from the study. There were no significant differences across any of the variables between those who participated and those who did not. Age was calculated from the date of birth to the day of the assessment. All girls and parents/guardians gave written informed consent and the project was approved by the Institutional Ethics Committee.

\section{Measures}

\section{Physical activity}

Physical activity was assessed using the self-report Physical Activity Questionnaire for Children (PAQ-C; Crocker, Bailey, Faulkner, Kowalski, \& McGrath, 1997). The PAQ-C is a 7-day selfreport recall instrument developed to assess general levels of physical activity during the school year. The PAQ-C provides a summary physical activity score derived from nine items, each scored on a five-point Likert scale where a score of 1 indicates low physical activity and a score of 5 indicates high physical activity. The PAQ-C has previously demonstrated acceptable validity and reliability in an early adolescent population (Welk \& Eklund, 2005) and the average reliability for the PAQ-C in this study was $\alpha=0.85$.

\section{Maturation}

Maturation was șelf-assessed using the Pubertal Development Scale (PDS; (Petersen, Crockett, Richards \& Boxer, 1988)). The PDS is a 5-item scale that assesses 5 physical changes in pubertal development. Pubertal development on each item is rated on a four-point scale. An overall pubertal score is calculated as a mean of the five items, to generate a four-stage score of development and was treated as a continuous variable.

\section{Physical self-perceptions}

Physical self-perceptions were assessed using the Children and Youth's Physical Self-Perception Profile (CY-PSPP; (Whitehead, 1995)) which is designed to assess self-perceptions within sub domains of the physical self. These are sport competence (Sport), physical condition (Condition), body attractiveness (Body) and muscular strength and development (Strength) and a fifth subscale measures overall physical self-worth (PSW). The sport competence subscale is drawn from Harter's (1982) Self-Perception Profile as this instrument had previously been validated with a similar age group (Whitehead, 1995). Each scale contains six items and the item score can range from 1 (low) to 4 (high) on a structured alternative scale, offering two opposing statements. The 
participant is first asked which of two statements best describes them and then decides whether it is really true or somewhat true of them. The CY-PSPP subscales have demonstrated acceptable internal reliability with young children and adolescents (Crocker et al., 2000; Welk \& Eklund, 2005). The average internal reliability estimates of the CY-PSPP subscales in this study were acceptable: Sport $(\alpha=0.82)$, Condition $(\alpha=0.81)$, Body $(\alpha=0.87)$, Strength $(\alpha=0.82)$ and $\operatorname{PSW}(\alpha=0.85)$.

\section{Physical data}

Body mass was assessed using a Seca precision dial scale (Seca, Vogel and Halke, Hamburg, Germany). Waist circumference was assessed using a steel flexible anthropometric tape (Rosscraft Innovations Inc, UK). Skinfolds were assessed using Harpenden skinfold calipers (ASSIST creative resources LTD) at five sites on the right side of the body; the bicep, tricep, subscapular, iliac crest and medial calf. Assessments were carried out according to the procedures identified by the International Society for the Advancement of Kinanthropometry (ISAK) and all intra-tester measurements reported had a Technical Error of Measurement (TEM) of less than 5\%.

\section{Procedures}

At baseline the researchers met with the participants at least 2 weeks prior to data collection in order to introduce the research project and staff and to distribute both parent and child consent forms. All participants completed the questionnaire in a group classroom setting and the questionnaire was split into 3 sections to allow a comfort break and to ensure the girls' attention was maintained. A full explanation for each section was provided and administration of the full questionnaire took an average of one hour. Following the questionnaire, the participants were taken in pairs to a private room to complete the physical measures where two researchers were always present with the girls. The measures took approximately $10-15 \mathrm{~min}$ per pair. Approximately 12 months later, data were collected using the same procedure.

\section{Data analysis}

All data were double imputed into SPSS version 12 (SPSS Inc., Chicago, IL, USA, version 12). The data were subsequently screened and checked for inconsistencies between the two spreadsheets. Participants for whom there were any missing variables were excluded, reducing the sample for analysis to 150 . Prior to further analyses, the data were tested for the assumptions of parametric tests. Although all of the variables did not meet these assumptions at either phase 1 or phase 2, subsequent log transformation of the data (Tabachnick \& Fidell, 2001) indicated no significant difference in the findings when using the log-transformed data or the original data. Therefore, to ease interpretation, parametric tests were used for subsequent data analysis on the original data (Tabachnick \& Fidell, 2001).

Paired sample t-tests were used to examine the changes in physical activity, maturation, physical characteristics and physical self-perceptions over the 12-month period. Cohen's $d$ effect sizes (Cohen, 1969) were calculated to measure the magnitude of the change in variables from phase 1 to phase 2. Intraclass correlation coefficients were calculated using a two-way mixed effect model to examine stability over the two phases. 
In order to determine whether (i) changes in maturation, physical characteristics and physical selfperceptions influenced change in physical activity and (ii) whether changes in maturation and physical characteristics influenced changes in physical self-perceptions, a two-step analytical strategy was used, consistent with Crocker et al. (2006). Firstly, the standardised residual or change score in all of the variables over the 12-months was determined using regression analysis (Field, 2005), with phase 1 being the independent variable and phase 2 being the dependent variable. The resulting residual change score reflects the degree of change from phase 1 to phase 2 (Sallis, Prochaska, Taylor, Hill, \& Geraci, 1999). Correlations between maturation, physical characteristics, physical selfperceptions and physical activity were examined and those variables significantly related to physical activity were entered into the regression. The physical self-worth variable was not included in the regression analysis since the four CY-PSPP subscales represent specific dimensions of the physical self and are collectively strong predictors of overall physical self-worth. Therefore, subsequent multiple regression analysis involved entry of the standardised residuals of only the four CYPSPP subscales at Step 1. This strategy allowed identification of key physical self-perception subscales that were associated with changes in physical activity over the 12-month period.

Correlations between maturation, physical characteristics and physical self- were examined and those significantly related to the physical self-perception variables were entered into the regression. Therefore, subsequent multiple regression analysis involved entry of the standardised residuals of body mass and sum of skinfolds at Step 1 (Field, 2005). This strategy allowed identification of key physical characteristics that were associated with changes in physical self-perceptions over the 12-month period.

\section{Results}

\section{Changes in physical activity, physical characteristics and physical self-perceptions}

Table 1 illustrates the means, standard deviations, effect sizes and intraclass correlation coefficients for physical activity, maturation, physical characteristics and the physical self-perceptions subscales for baseline (phase 1 ) and phase 2 data. The mean physical activity levels were significantly lower in phase $2(2.78 \pm 0.57)$ than in phase $1(3.06 \pm 0.71),(p<0.01)$ and this effect size was medium. The participants increased significantly in all of the physical characteristics from phase 1 to phase 2 with varying effect sizes for group mean changes, with the largest effect size for body mass. There was a significant increase in their maturational status, with a large effect size. There were overall significant decreases from phase 1 to phase 2 on the CY-PSPP subscales of body attractiveness $(p<0.05)$ and physical self-worth $(p<0.01)$ and these effect sizes were relatively small.

\section{Influence of maturation, physical characteristics and physical self-perceptions on the change in physical activity}

The correlations among standardised residual change scores for physical activity, maturation, physical characteristics and physical self-perceptions are shown in Table 2. Change in maturation or physical characteristics was not associated with the change in physical activity. However correlations indicated that physical activity change was associated with change in all of the Children 
Table 1

Descriptive statistics for physical activity, maturation, physical characteristics and physical self-perceptions at phase 1 and phase 2

\begin{tabular}{lllll}
\hline & Phase $1(N=150)$ & Phase 2 $(N=150)$ & $d$ & ICC \\
\hline PAQ-C score & $3.06 \pm 0.71$ & $2.78 \pm 0.57$ & $0.40^{* *}$ & 0.50 \\
PDS score & $1.61 \pm 0.69$ & $2.10 \pm 0.64$ & $0.71^{* *}$ & 0.59 \\
Body mass (kg) & $42.9 \pm 8.80$ & $48.9 \pm 9.9$ & $0.68^{* *}$ & 0.94 \\
Waist circumference (cm) & $63.9 \pm 6.7$ & $65.7 \pm 8.7$ & $0.27^{* *}$ & 0.77 \\
Sum of skinfolds (mm) & $66.3 \pm 24.6$ & $77.2 \pm 29.5$ & $0.44^{* *}$ & 0.87 \\
Sport competence $^{\mathrm{a}}$ & $2.79 \pm 0.61$ & $2.76 \pm 0.60$ & 0.05 & 0.66 \\
Body attractiveness $^{\mathrm{a}}$ & $2.53 \pm 0.66$ & $2.42 \pm 0.67$ & $0.17^{*}$ & 0.63 \\
Physical condition $^{\mathrm{a}}$ & $2.79 \pm 0.57$ & $2.76 \pm 0.60$ & 0.05 & 0.67 \\
Physical strength $^{\mathrm{a}}$ & $2.50 \pm 0.56$ & $2.52 \pm 0.54$ & $0.29^{* *}$ & 0.69 \\
Physical self-worth $^{\mathrm{a}}$ & $2.78 \pm 0.63$ & $2.60 \pm 0.65$ & & 0.57 \\
\hline
\end{tabular}

*Significant difference between phase 1 and phase $2, p<0.05$.

**Significant difference between phase 1 and phase $2, p<0.01 ; d$ is the reported effect size; ICC is the single measure intraclass correlation.

PAQ-C: Physical Activity Questionnaire for Children ( $\max$ score $=5)$; PDS: Pubertal Development Scale $(\max =4)$.

${ }^{\text {a }}$ CY-PSPP (Children and Youth's Physical Self-Perception Profile) subscales (max score $=4$ ).

and Youth's Physical Self-Perception Profile (CY-PSPP) subscales, with sport competence being the dominant correlate $(r=0.31)$. Multiple regression analysis indicated that the physical selfperception subscales accounted for $9.9 \%$ of the explained variance in physical activity change. In the model, only the physical self-perception subscale of physical condition was a significant individual predictor of physical activity (Table 3).

\section{Influence of maturation and physical characteristics on the change in physical self-perceptions}

Correlations indicated that change in sum of skinfolds and body mass was negatively associated with change in the CY-PSPP subscales of body attractiveness and physical self-worth.

Table 2

Pearson correlation coefficients for physical activity, maturation, physical characteristics and physical self-perceptions change standardised residuals

\begin{tabular}{|c|c|c|c|c|c|c|c|c|c|c|}
\hline Variable & 1 & 2 & 3 & 4 & 5 & 6 & 7 & 8 & 9 & 10 \\
\hline 1. PAQ-C score & - & & & & & & & & & \\
\hline 2. PDS score & -0.13 & - & & & & & & & & \\
\hline 3. Body mass $(\mathrm{kg})$ & 0.00 & -0.02 & - & & & & & & & \\
\hline 4. Waist circumference $(\mathrm{cm})$ & -0.01 & -0.08 & $-0.42 *$ & - & & & & & & \\
\hline 5. Sum of skinfolds & -0.05 & -0.02 & $-0.46^{*}$ & -0.15 & - & & & & & \\
\hline 6. Sport competence ${ }^{a}$ & $0.31^{*}$ & -0.06 & 0.12 & 0.08 & -0.09 & - & & & & \\
\hline 7. Body attractiveness ${ }^{\mathrm{a}}$ & 0.17 & -0.04 & $0.21^{*}$ & -0.10 & $-0.22 *$ & $0.32 *$ & - & & & \\
\hline 8. Physical condition ${ }^{\mathrm{a}}$ & $0.29 *$ & -0.09 & 0.13 & 0.07 & -0.06 & $0.53^{*}$ & $0.26^{*}$ & - & & \\
\hline 9. Physical strength ${ }^{\mathrm{a}}$ & $0.20 *$ & 0.07 & 0.09 & 0.03 & -0 & $0.55^{*}$ & $0.42 *$ & $0.53 *$ & - & \\
\hline 10. Physical self-worth ${ }^{\mathrm{a}}$ & $0.28^{*}$ & -0.07 & 0.17 & -0.09 & $-0.22 *$ & $0.42 *$ & $0.71^{*}$ & $0.35^{*}$ & $0.52 *$ & - \\
\hline
\end{tabular}

*Significance $(p<0.05)$.

PAQ-C: Physical Activity Questionnaire for Children $(\max =5)$; PDS: Pubertal Development Scale $(\max =4)$.

${ }^{a}$ CY-PSPP (Children and Youth's Physical Self-Perception Profile) subscales (max score $=4$ ). 
Table 3

Multiple regression analysis examining predictors of change in physical activity

\begin{tabular}{|c|c|c|c|c|}
\hline Step 1 & Variable & $B$ & SE $B$ & $\beta$ \\
\hline \multirow[t]{5}{*}{1} & Physical self-perceptions & & & \\
\hline & Sport competence & 0.203 & 0.108 & 0.189 \\
\hline & Body attractiveness & 0.059 & 0.093 & 0.057 \\
\hline & Physical condition & 0.203 & 0.101 & $0.200^{*}$ \\
\hline & Physical strength & -0.037 & 0.105 & -0.036 \\
\hline
\end{tabular}

Note: $R^{2}=0.122 ; R^{2} \Delta=0.099(p \mathrm{~s}<0.05) .{ }^{*} p<0.05$.

Multiple regression analysis indicated that the physical characteristics of sum of skinfolds and body mass accounted for $8.9 \%$ of the explained variance in the change of perceptions of body attractiveness and $6.9 \%$ of the explained variance in the change of overall physical self-worth. In the model, only body mass was a significant individual predictor of perceptions of body attractiveness and physical self-worth (Tables 4 and 5).

\section{Discussion}

The purpose of this study was to examine the influence of maturation on physical self-perceptions and the relationship with physical activity in adolescent girls over 12 months. Consistent with a number of studies that have reported a decline in physical activity during adolescence (Aaron et al., 2002; Bromley et al., 2003; Kimm et al., 2002; Nelson et al., 2006), there was a significant decrease in overall physical activity levels over the 12 months. Recently, Davison et al. (2007) found similar decreases in physical activity in adolescent girls of similar chronological age to the population in the present study. Additionally, this drop in physical activity is comparable to previous research using the Physical Activity Questionnaire for Children (PAQ-C) to examine change in adolescents' physical activity levels (Crocker et al., 2003, 2006). This drop in physical activity levels over 12 months further emphasises the physical inactivity issue within this population and the need to develop appropriate interventions to prevent this decrease.

As expected, the participants advanced in maturational status and all of the physical characteristics significantly increased over the 12 months. These findings would suggest that the onset of maturation does lead to an increase in physical characteristics related to body size, as previously documented (Malina et al., 2004). Perceptions of body attractiveness and physical self-worth were significantly less positive after the 12 months, indicating that aspects of the physical self become less positive with age in early adolescent girls. This finding is consistent with previous research highlighting a decrease in physical self-perceptions with age in early adolescents (Hagger, Biddle

Table 4

Multiple regression analysis examining predictors of change in perceptions of body attractiveness

\begin{tabular}{lllll}
\hline Step 1 & Variable & $B$ & SE $B$ & $\beta$ \\
\hline 1 & Body mass & -0.276 & 0.094 & $-0.276^{*}$ \\
& Sum of skinfolds & -0.070 & 0.095 & -0.069 \\
\hline
\end{tabular}

Note: $R^{2}=0.102 ; R^{2} \Delta=0.089(p \mathrm{~s}<0.05) .{ }^{*} p<0.05$. 
Table 5

Multiple regression analysis examining predictors of change in physical self-worth

\begin{tabular}{lllll}
\hline Step 1 & Variable & $B$ & SE $B$ & $\beta$ \\
\hline 1 & Body mass & -0.230 & 0.096 & $-0.228^{*}$ \\
& Sum of skinfolds & -0.091 & 0.097 & -0.089 \\
\hline
\end{tabular}

Note: $R^{2}=0.082 ; R^{2} \Delta=0.069(p \mathrm{~s}<0.05) .{ }^{*} p<0.05$.

\& Wang, 2005). Previous research by Crocker et al. $(2003,2006)$ reported significant decreases in perceptions of sport competence, physical condition and physical strength over 24 months in adolescent girls aged 14-17 years. The differences in research findings could be explained by marked age differences in the participants in each study, possibly indicating that different aspects of the physical self change at different times during the transitional period of adolescence. Crocker et al. (2006) does suggest that a focus on examining the physical self-perceptions of early adolescent girls may be more appropriate in understanding changing physical activity behaviour. Therefore, monitoring physical self-perceptions longitudinally is worthwhile during adolescence, especially considering the important role physical self-perceptions have in understanding PA behaviour (Fox, 1997).

Despite an increase in physical characteristics, the decline in physical activity levels over 12 months was not influenced by maturational status or physical characteristics. These findings are in line with early research by Armstrong et al. (1990) whose findings suggested that maturation had no influence on sustained periods of physical activity in both boys and girls. However, our research findings are contrary to more recent research evidence (Baker et al., 2007; Thompson et al., 2003). In particular, Baker and colleagues (Baker et al.) identified that earlier timing of pubertal development, relative to peers, at age 11 years is associated with lower levels of physical activity at age 13 years, independent of pre-established PA levels or body fat. However, there are methodological differences between that and the present study which could to some extent explain the different research findings. Baker and colleagues used a combination of objective and subjective measures to classify maturational status compared to our sole use of the self-report Pubertal Development Scale (PDS). Furthermore, the participants were classified as early-maturers or latematurers relative to their peers unlike this study which used a direct measure of maturation. This could suggest that maturation relative to peers is more influential on physical activity behaviour than actual maturational status, however further research is needed to explore this possibility.

Another probable explanation for the lack of maturational influence on the change in physical activity levels could be due to limited advancement through the maturational process over the 12 months. Although the effect of the changes in maturational status over 12 months was large, it remains questionable whether 12 months during early adolescence is a sufficient time-period for the physical characteristics accompanying maturation to develop and subsequently influence physical activity behaviour.

The results examining the influence of physical self-perceptions on the change in physical activity emphasise the role the physical self has to play in the adoption and maintenance of physical activity behaviour. In the present study, the resulting change in perceptions of sport competence, physical condition and physical strength over the 12 months were shown to be significant positive correlates of the change in physical activity. Additionally, the explained variance in 
physical activity change was partially accounted for by physical self-perceptions whilst physical condition was shown to be a significant individual predictor of physical activity change. Physical condition consists of perceptions of condition, stamina, fitness, ability to maintain exercise and confidence in an exercise setting. This is contrary to Sport competence which consists of perceptions of confidence in sport and the ability to learn sport (Fox, 1997). Therefore, it could be argued that aspects of the physical self which are most influential on the decrease in physical activity in early adolescent girls are related to perceptions of condition, fitness and confidence in exercise settings rather than sport settings. Therefore suggesting that interventions aimed at increasing physical activity in early adolescent girls should focus on increasing perceptions of condition, fitness and confidence in exercise settings.

These findings are consistent with recent longitudinal research by Crocker et al. $(2003,2006)$ who assessed the relationship between the physical self-concept and physical activity in adolescent girls with $12.9 \%$ of the explained variance in physical activity change accounted for by physical self-perceptions. In addition, physical condition was the only significant individual predictor of PA change over 3 years. However, it is important to note that in both the present study and findings by Crocker et al. $(2003,2006)$, the variance in physical activity accounted for by physical self-perceptions was relatively small and other factors related to this drop in physical activity need to be considered longitudinally.

The process of maturation is important in the formation of the physical self during adolescence, with the physical changes that accompany maturation possibly leading to an increase in body dissatisfaction as girls move away from the cultural female ideal of a thin and slender body (Rodin, Silberstein, \& Striegal-Moore, 1985). Despite results indicating that maturation had no direct influence on the change in physical self-perceptions, the change in the physical characteristics of body mass and fat mass, as assessed by sum of skinfolds, were shown to be significant negative correlates of the change in perceptions of body attractiveness and physical self-worth. In addition, body mass was shown to be a significant individual predictor of the change in perceptions of body attractiveness and physical self-worth. Therefore, it could be argued that although maturation may not have a direct influence on physical self-perceptions, the increase in body mass normally associated with maturation could be associated with aspects of the physical self becoming less positive in adolescent girls. However, these aspects of the physical self that were influenced by changes in physical characteristics were not the same aspects of the physical self which were most influential on the decrease in physical activity. Therefore, our findings suggest that in early adolescent girls, there is limited evidence of a direct link between the physical changes associated with maturation, changes in physical self-perceptions and the decrease in physical activity. However this may change as girls progress through adolescence and the maturational process, and further longitudinal research would be of value.

The strength of this study is that it used a longitudinal design to examine the influence of maturation on physical self-perceptions and the relationship with physical activity behaviour in an early adolescent population where physical self-perceptions are relatively unstable (Crocker et al., 2006). One limitation of the study is the reliance on self-report data to measure both physical activity and maturation, which could be influenced by accuracy of recall of physical activity information and social desirability influences. Additionally, a greater number of data time points should be considered for future research as the majority of the girls in the study were only partially through the maturational process. 
In conclusion, this study highlights that in early adolescent girls, maturation has a limited influence on the physical activity behaviours of early adolescent girls. However, there was evidence that the increase in body mass associated with maturation was related to perceptions of body attractiveness and physical self-worth becoming less positive. In addition, decreases in physical self-perceptions partially accounted for the decrease in physical activity over the 12 months. Recent research by Davison et al. (2007) examined possible psychological mechanisms to explain the association between early pubertal development and declines in physical activity among adolescent girls. However, from the findings of the present study, it is difficult to establish a link to understand the coincidental decrease in physical activity levels and advancement in maturational status during adolescence. The finding that physical activity levels declined considerably over 12 months in early adolescent girls in itself warrants considerable attention and although this variance in physical activity was partly accounted for by physical self-perceptions, this was a relatively small contribution and other factors related to this drop in physical activity needs to be considered longitudinally. For example, the influence of social and cultural variables, physical environment variables and behavioural variables could be considered in order to understand physical activity behaviour in this population and furthermore, to inform appropriate and effective intervention practices.

\section{Acknowledgements}

The researchers would like to acknowledge the enthusiasm and assistance of the staff and pupils from the schools involved in this project and the support from sportscotland, particularly Kristi Long and Jessica Lindhof.

\section{References}

Aaron, D. J., Storti, K. L., Robertson, R. J., Kriska, A. M., \& LaPorte, R. E. (2002). Longitudinal study of the number and choice of leisure time physical activities from mid to late adolescence: implications for school curricula and community recreation programs. Archives of Pediatrics and Adolescent Medicine, 156(11), $1075-1080$

Armstrong, N., Balding, J., Gentle, P., \& Kirby, B. (1990). Patterns of physical activity among 11 to 16 year old British children. British Medical Journal, 301(6745), 203-205.

Baker, B. L., Birch, L. L., Trost, S. G., \& Davison, K. K. (2007). Advanced pubertal status at age 11 and lower physical activity in adolescent girls. Journal of Pediatrics, 151(5), 488-493.

Biddle, S. J., Whitehead, S. H., O’Donovan, T. M., \& Nevill, M. E. (2005). Correlates of participation in physical activity for adolescent girls: a systematic review of recent literature. Journal of Physical Activity and Health, 2, 423-434.

Bromley, C., Chaudhury, M., Craig, R., Deverill, C., Erens, B., Fuller, E., et al. (2003). Scottish health survey: Children's report. Edinburgh: Scottish Executive.

Cohen. (1969). Statistical power for the behavioural sciences (2nd ed.). New York: Academic Press.

Crocker, P. R., Bailey, D. A., Faulkner, R. A., Kowalski, K. C., \& McGrath, R. (1997). Measuring general levels of physical activity: preliminary evidence for the physical activity questionnaire for older children. Medicine and Science in Sports and Exercise, 29(10), 1344-1349.

Crocker, P. R., Eklund, R. C., \& Kowalski, K. C. (2000). Children's physical activity and physical self-perceptions. Journal of Sports Sciences, 18(6), 383-394. 
Crocker, P., Sabiston, C., Forrestor, S., Kowalski, N., Kowalski, K., \& McDonough, M. (2003). Predicting change in physical activity, dietary restraint, and physique anxiety in adolescent girls: examining covariance in physical selfperceptions. Canadian Journal of Public Health, 94(5), 332-337.

Crocker, P., Sabiston, C., Kowalski, K., McDonough, M., \& Kowalski, N. (2006). Longitudinal assessment of the relationship between physical self-concept and health-related behaviour and emotion in adolescent girls. Journal of Applied Sport Psychology, 18, 185-200.

Davison, K. K., Werder, J. L., Trost, S. G., Baker, B. L., \& Birch, L. L. (2007). Why are early maturing girls less active? Links between pubertal development, psychological well-being, and physical activity among girls at ages 11 and 13 . Social Science and Medicine, 64(12), 2391-2404.

Department of Health. (2004). At least five a week: Evidence of the impact of physical activity and its relationship to health. UK: Department of Health.

Field, A. (2005). Discovering statistics using SPSS (2nd ed.). London: Sage Publications.

Fox, K. R. (1997). The physical self: From motivation to well-being. Champaign, II: Human Kinetics.

Grunbaum, J., Kann, L., Kinchen, S., Ross, J., Hawkins, J., \& Lowry, R. (2004). Youth risk behaviour surveillance United States 2003. Morbidity and Mortality Weekly, 53(SS-2), 1-96.

Hagger, M. S., Biddle, S., \& Wang, C. K. (2005). Physical self-concept in adolescence: generalisability of a multidimensional, hierarchical model across gender and grade. Educational and Psychological Measurement, 65(2), $297-322$.

Harter, S. (1982). The perceived competence scale for children. Child Development, 53, 87-97.

Harter, S. (1999). The construction of the self. New York: Guilford.

Horn, T. S. (2004). Developmental perspectives on self-perceptions in children and adolescents. In M. Weiss (Ed.), Developmental sport and exercise psychology: A lifespan perspective. West Virginia: Fitness Information Technology, Inc.

Kimm, S. Y., Glynn, N. W., Kriska, A. M., Barton, B. A., Kronsberg, S. S., Daniels, S. R., et al. (2002). Decline in physical activity in black girls and white girls during adolescence. New England Journal of Medicine, 347(10), $709-715$.

Malina, R. M., Bouchard, C., \& Bar-Or, O. (2004). Growth, maturation and physical activity (2nd ed.). Human Kinetics.

Nelson, M. C., Neumark-Stzainer, D., Hannan, P. J., Sirard, J. R., \& Story, M. (2006). Longitudinal and secular trends in physical activity and sedentary behavior during adolescence. Pediatrics, 118(6), 1627-1634.

Petersen, A. C., Crockett, L., Richards, M., \& Boxer, A. (1988). A self-report measure of pubertal status: reliability, validity and initial norms. Journal of Youth and Adolescence, 17(2), 117-133.

Raustorp, A., Stahle, A., Gudasic, H., Kinnunen, A., \& Mattsson, E. (2005). Physical activity and self-perception in school children assessed with the children and youth - physical self-perception profile. Scandinavian Journal of Medicine and Science in Sports, 15(2), 126-134.

Rodin, J., Silberstein, L. R., \& Striegel-Moore, R. H. (1985). Women and weight: A normative discontent. Lincoln, NE: University of Nebraskao.

Sallis, J. F., Prochaska, J. J., Taylor, W. C., Hill, J. O., \& Geraci, J. C. (1999). Correlates of physical activity in a national sample of girls and boys in grades 4 through 12. Health Psychology, 18(4), 410-415.

Smith, A. L. (2004). Measurement of social physique anxiety in early adolescence. Medicine and Science in Sports and Exercise, 36(3), 475-483.

Sonstroem, R. J. (1997). The psychological benefits of exercise. Medicine and Health Rhodes Island, 80(9), $295-296$.

Tabachnick, B. G., \& Fidell, L. S. (2001). Using multivariate statistics. Needham heights, MA: Pearson Education Company.

Thompson, A. M., Baxter-Jones, A. D., Mirwald, R. L., \& Bailey, D. A. (2003). Comparison of physical activity in male and female children: does maturation matter? Medicine and Science in Sports and Exercise, 35(10), 1684-1690.

Weiss, M., Bredemeier, B. J., \& Shewchuck, R. M. (1986). The dynamics of perceived competence, perceived control and motivational orientation in youth sport. In M. Weiss, \& D. Gould (Eds.), Sport for children and youths (pp. 89-101). Champaign, IL: Human Kinetics.

Weiss, M. R., \& Horn, T. S. (1990). The relation between children's accuracy estimates of their physical competence and achievement-related characteristics. Research Quarterly for Exercise and Sport, 61(3), 250-258.

Welk, G. J., \& Eklund, B. (2005). Validation of the children and youth physical self-perception profile for young children. Psychology of Sport and Exercise, 6, 51-65.

Whitehead, J. R. (1995). A study of children's physical self-perceptions using an adapted physical self-perception profile questionnaire. Pediatric Exercise Science, 7, 132-151. 